\title{
PIERCING EM UNIVERSITÁRIOS E SUA ASSOCIAÇÃO COM BEBER EM BINGE E USO DE DROGAS ILÍCITAS
}

\section{Samuel Santos Souza ${ }^{1}$; Matheus Cordeiro Fonseca ${ }^{2}$; Paula Cristina Pelli Paiva ${ }^{3}$; Haroldo Neves de Paiva ${ }^{4}$; Paulo Messias de Oliveira Filho ${ }^{5}$}

1Pós-Graduando em Imunologia e Parasitologia Básicas e Aplicadas, Universidade Federal de Mato Grosso (UFMT), Barra do Garças, Mato Grosso.

${ }^{2}$ Graduando em Odontologia, Universidade Federal dos Vales do Jequitinhonha e Mucuri (UFVJM), Diamantina, Minas Gerais.

${ }^{3,4,5}$ Doutor, Universidade Federal dos Vales do Jequitinhonha e Mucuri (UFVJM), Diamantina, Minas Gerais.

\section{DOI: 10.47094/IICNNESP.2021/58}

\section{RESUMO}

Objetivo avaliar associação entre o uso de piercing e beber em binge e uso de drogas ilícitas por universitários da área da Saúde. Estudo transversal com amostra de 190 universitários. O curso foi selecionado por sorteio e os alunos por conveniência. Foi questionado ao universitário sobre o uso de piercing e a resposta foi dicotomizada, 0 para que nunca fez uso e 1 já fez e faz uso. Os instrumentos The Alcohol Use Disorders Identification Test C (AUDIT-C) e Teste para Triagem do Envolvimento com Álcool, Cigarro e Outras Substâncias (ASSIST) foram utilizados para avaliar o consumo de álcool e drogas ilícitas. Foram realizadas análises de frequências e testes de associação $(p<0,05)$. A prevalência do piercing foi de $46,8 \%(\mathrm{n}=89)$ e esteve associado estatisticamente ao sexo feminino $(\mathrm{p}=0,016)$, beber em Binge $(\mathrm{p}=0,011)$ e drogas ilícitas $(\mathrm{p}=0,001)$.

PALAVRAS-CHAVE: Universidade. Etilismo. Alunos.

ÁREA TEMÁTICA: Condições Sociais e de Saúde

\section{INTRODUÇÃO}

O piercing é uma prática que consiste em perfurar partes do corpo, para inserir anéis, brincos e piercings. Usado como uma forma de decoração do corpo, tanto para fins puramente estéticos, ritualísticos, ou para afirmar que alguém pertence a uma classe ou grupo étnico específico. Embora o piercing tenha se tornado uma tendência dominante, pode estar associado a complicações médicas e comportamentos de alto risco (COVELLO et al., 2020).

Além disto, a literatura cita vários comportamentos de risco associados aos portadores de piercing, como comportamento alimentar desordenado, suicídio, uso de drogas ilícitas, depressão, uso de fumo, álcool, abuso de drogas e atividade sexual de risco (CARROLL et al., 2002). 
Assim, o objetivo do estudo foi sobre a prevalência do piercing e fatores associados entre os estudantes universitários de um curso da área de saúde.

\section{METODOLOGIA}

Foi realizado um estudo transversal com uma amostra de 190 universitários, matriculados do primeiro ao nono período de curso da área da saúde da Universidade Federal dos Vales do Jequitinhonha e Mucuri. O curso da saúde foi selecionado por sorteio e os universitários por conveniência. O projeto foi aprovado pelo Comité de Ética em Pesquisa (parecer: 79177617.1.0000.5108). Foi questionado ao graduando quanto ao usou de piercing e as respostas dicotomizadas em 0 para que nunca fez uso e 1 já fez e faz uso. Para avaliar o consumo de bebida alcoólica em binge e o uso de drogas ilícitas foram adotados os instrumentos The Alcohol Use Disorders Identification Test C (AUDIT-C) e Teste para Triagem do Envolvimento com Álcool, Cigarro e Outras Substâncias (ASSIST), respectivamente. Todas as demais drogas deste instrumento, com exceção de bebidas alcoólicas e tabaco, foram consideradas como drogas ilícitas, e não foram computadas isoladamente. $O$ uso de drogas foi avaliado por meio da primeira pergunta do questionário ASSIST: você já fez uso na vida de drogas ilícitas? Com opção de resposta 0 para não e 1 para sim. Os dados foram avaliados por meio do programa Statistical Package for the Social Sciences (SPSS for Windows, version 20.0, SPSS Inc, Chicago, IL, USA) e incluiu distribuição de frequência e testes de associação.

\section{RESULTADOS E DISCUSSÕES}

A prevalência do piercing foi de 46,8\% $(\mathrm{n}=89)$. Piercing foi significantemente associado com sexo feminino $(p=0,016)$, beber em Binge $(p=0,011)$ e drogas ilícitas $(p=0,001)$. Controlado pelo sexo, os universitários com piercing tiveram chances 2,5 vezes maiores de beber em binge [OR $=2,5($ IC95\%:1,20-4,88) $\mathrm{p}=0,006]$ e quase 4 vezes mais chances de pertencer ao grupo que usaram droga ilícita $[\mathrm{OR}=3,9(\mathrm{IC} 95 \%: 1,83-8,25) \mathrm{p}=0,000]$.

A alta prevalência de piercing (47,3\%) em adultos jovens observada neste estudo foi superior a maioria dos estudos cuja prevalência variou de 0,8\% a 42\% (CARROLL et al., 2002; BROOKS et al., 2003; HENNEQUIN-HOENDERDOS, SLOT, VAN DER WEIJDEN, 2012). Em relação ao sexo, foi observada uma prevalência maior nas mulheres (80,9\%), e um resultado semelhante foi observado em uma importante revisão sistemática da literatura, onde a prevalência de piercings foi aproximadamente quatro vezes maior entre as mulheres quando comparada com homens $(5,6 \%$ mulheres versus 1,55 homens) (HENNEQUIN-HOENDERDOS, SLOT, VAN DER WEIJDEN, 2012).

O uso do piercing, beber em binge e o uso de drogas ilícitas mantiveram-se associados independente do sexo $(p=0,005)$. Escassos são os estudos que investiguem esta associação. Indivíduos com tatuagens e piercings mostraram uma maior propensão a comportamentos sexuais de risco e abuso de drogas (CARROLL et al., 2002, ARMSTRONG et al., 2007) uso de fumo e álcool (BOSELLO et al., 2010). 


\section{CONCLUSÃO}

O presente estudo trouxe informações importantes sobre o uso de piercing em universitários de curso da área de saúde, uma vez que profissionais de saúde, estão em uma posição ideal para oferecer informações sobre o uso adequado de piercings. Contribuiu também com informações sobre o uso de drogas ilícitas e padrão de consumo de álcool em universitários que usam e não usam o piercing, assunto este ainda escasso em trabalhos desta natureza, e a enxergar o piercing não somente como uma adorno, mas como também um possível problema de saúde pública.

\section{PRINCIPAIS REFERÊNCIAS}

ARMSTRONG, M.L.; KOCH, J.R.; SAUNDERS, J.C. ROBERTS, A. E., \& OWEN, D. C. The hole picture: risks, decision making, purpose, regulations, and the future of body piercing. Texas: Clinical Dermatology, 2007.

BOSEllO, R.; FAVARO, A.; ZANETTI, T.; SOAVE, M.; VIDOTTO, G.; HUON, G.; SANTONASTASO, P. Tattoos and piercings in adolescents: family conflicts and temperament. Padova: Revist of Psichiatric, 2010.

BROOKS, T. L., WOODS, E. R., KNIGHT, J. R., SHRIER, L. A. Body modification and substance use in adolescents: is there a link? Boston: Journal of Adolescent Health, 2003.

CARROL, S.T.; RIFFENBURGH, R. H.; ROBERTS, T. A.; MYHRE, E.B. Tattoos and body piercings as indicators of adolescent risk-taking behaviors. San Diego: Pediatrics, 2002.

COVELlO, F.; SALERNO, C.; GIOVANNINI, V.; CORRIDORE, D.; OTTOLENGHI, L.; VOZZA, I.

Piercing and Oral Health: A Study on the Knowledge of Risks and Complications. Roma: International Journal Environent Research Public Health, 2020.

HENNEQUIN-HOENDERDOS, N.L.; SLOT, D.E.; VAN DER WEIJDEN, G.A. The prevalence of oral and peri-oral piercings in young adults: a systematic review. Amsterdam: International of Journal Dentistry Hygienic, 2012.

HENRIQUE, I.F.S.; DE MICHELI, D.; LACERDA, R.B.; FORMIGONI, M.L.O.S. Validação da versão brasileira do Teste de Triagem do Envolvimento com Álcool, Cigarro e outras Substâncias (ASSIST). São Paulo: Revista da Associação Médica Brasileira, 2004.

MENESES-GAYA, C.; ZUARDI, A.W.; LOUREIRO, S.R.; HALLAK, J. E. C.; TREZNIAK, C.; MARQUES, J. M. A. M; SOUSA, J. P. M; CHAGAS, M. H. N.; SOUZA, M. S.; CRIPPA, J. A. S. Is the full version of the Audit really necessary? Study of the validity and internal construct of its abbreviated versions. Ribeirão Preto: Alcohol Clinical Express Research, 2010. 